\title{
More than FOLFOX and FOLFIRI: The Management of Metastatic Colorectal Cancer in the Era of Precision Oncology
}

My chosen article for the Editor's Pick in this issue is 'More than FOLFOX and FOLFIRI: Management of Metastatic Colorectal Cancer in the Era of Precision Oncology' by Jácome and Johnson. The paper discusses the current landscape and standard of care for metastatic colorectal cancer (mCRC), a disease known for its heterogeneity and poor prognosis. Enhancements in molecular biology in relation to oncology have allowed the identification of specific molecular subtypes and novel therapeutic targets. In the review, Jacome and Johnson describe the current and emerging predictive biomarkers in $\mathrm{mCRC}$ and present landmark clinical trials that have allowed for evolving and improving precision in therapeutic management of the disease. Promising findings with targeted therapies offer the possibility of a new era of precision oncology and personalised treatments sustaining hope for patients with mCRC.
Authors:
Alexandre A. Jácome, ${ }^{1}$ Benny Johnson ${ }^{2}$
1. Department of Gastrointestinal Medical Oncology, Oncoclínicas, Belo Horizonte, Minas Gerais, Brazil
2. Department of Gastrointestinal Medical Oncology, Division of Cancer Medicine, The University of Texas MD Anderson Cancer Center, Houston, Texas, USA
*Correspondence to bjohnson6@mdanderson.org

Disclosure:

The authors have declared no conflicts of interest.

Received:

03.06.21

Accepted:

23.08.21

Citation:

EMJ Oncol. 2021;9[1]:43-52.

\section{Abstract}

Metastatic colorectal cancer (mCRC) is a markedly heterogeneous disease, which portends a poor prognosis, with an estimated 5-year overall survival rate of approximately $15 \%$. The standard of care of systemic therapy remains fluoropyrimidine-based chemotherapy, with modest results, despite improvements with the combination with anti-angiogenics and anti-epidermal growth factor receptor therapy.

Significant advances in cancer therapy have been observed in the past two decades. The enhanced appreciation of molecular biology in oncology has allowed for the identification of specific molecular subtypes and novel therapeutic targets. Nevertheless, meaningful precision-based advancements in the therapeutic options for $\mathrm{mCRC}$ have been challenging and slow to realisation. Comprehensive molecular profiling and circulating tumour DNA highlight a heterogeneous disease at the genomic, epigenomic, and transcriptomic levels, and with a low frequency of actionable alterations. 
In the present review, the authors describe the current and emerging predictive biomarkers in $\mathrm{mCRC}$, as well as present landmark clinical trials that have allowed for evolving precision in the therapeutic management. The understanding of the benefit of immune checkpoint inhibitors in patients with high microsatellite instability cancer and in those with POLE mutations or high tumour mutational burden, the combination of BRAF with epidermal growth factor receptor inhibition in BRAF V600-mutated patients, the use of allele-specific KRAS G12C inhibitors, the promising findings of dual anti-HER2 therapy in HER2-positive $\mathrm{mCRC}$, and the possibility to offer targeted therapy for patients harbouring gene fusions NTRK/ALK/ROS1 have ushered in a new era of precision oncology for mCRC, providing personalised treatments and sustaining hope for patients affected by this challenging disease.

\section{INTRODUCTION}

Colorectal cancer (CRC) is the second most common cause of cancer-related death in the USA. ${ }^{1}$ Despite improvements in screening rates and in the overall survival (OS) of patients with localised and advanced disease over the past few decades, the 5-year OS of patients with metastatic disease is still extremely poor and estimated to be approximately $15 \% .^{2,3}$

Significant advances in cancer therapy have been observed in the past two decades. The enhanced appreciation of molecular biology in oncology has allowed for the identification of specific molecular subtypes and novel therapeutic targets. This era of precision oncology allows for the development of biomarker-guided therapeutics and has markedly transformed the landscape of cancer treatment. Precision medicine represents a paradigm shift in oncology, moving from a histology-based chemotherapy to include genome-specific targeted therapy, which has promoted ongoing discovery for novel biomarkers in all malignancies.

Nevertheless, the emergence of precision oncology has drastically improved the management of CRC. Comprehensive molecular profiling confirms a markedly heterogeneous disease at the genomic, epigenomic, and transcriptomic levels, but currently with low frequency of actionable alterations. For more than a decade, personalised therapy in CRC was restricted to the identification of RAS mutations, which are predictive markers of resistance to antiepidermal growth factor receptor (EGFR) monoclonal antibodies. Only recently, clinical trials addressing novel genome-guided personalised therapies in specific molecular subtypes of CRC have been successfully completed, expanding the clinical relevance of precision oncology in CRC.

In this review, the authors describe the current and emerging predictive biomarkers in metastatic CRC ( $\mathrm{mCRC}$ ), as well as present landmark clinical trials that have allowed for evolving precision in the management of this heterogenous disease.

\section{ANTI-EPIDERMAL GROWTH FACTOR RECEPTOR MONOCLONAL ANTIBODIES IN METASTATIC COLORECTAL CANCER IN THE PAST TWO DECADES: PRECISION THAT NEEDS FURTHER REFINEMENT}

For almost two decades, the clinical applicability of precision in $\mathrm{MCRC}$ has been limited to the use of the anti-EGFR monoclonal antibodies cetuximab and panitumumab for RAS wild-type disease. The knowledge accumulated over the past 20 years has demonstrated that the benefit offered by these monoclonal antibodies is restricted to a smaller subset of $\mathrm{MCRC}$ than initially proposed (Figure 1). Pure predictive biomarkers reflecting patient specific sensitivity to anti-EGFR monoclonal antibodies remains a developing area in $\mathrm{mCRC}$. Interestingly, evidence accumulated over the past few years suggest that not only expanded RAS mutations such as NRAS and HRAS may confer additional resistance to cetuximab or panitumumab but also BRAF, PIJKCA, HER2, MET, $P T E N$, and AKT1 abnormalities, as well as NTRK/ ROS1/ALK/RET rearrangements. ${ }^{4-9}$ Furthermore, recent studies have consistently demonstrated that patients with right-sided tumours derive lower, if any, benefit from that therapy. ${ }^{10,11}$ 


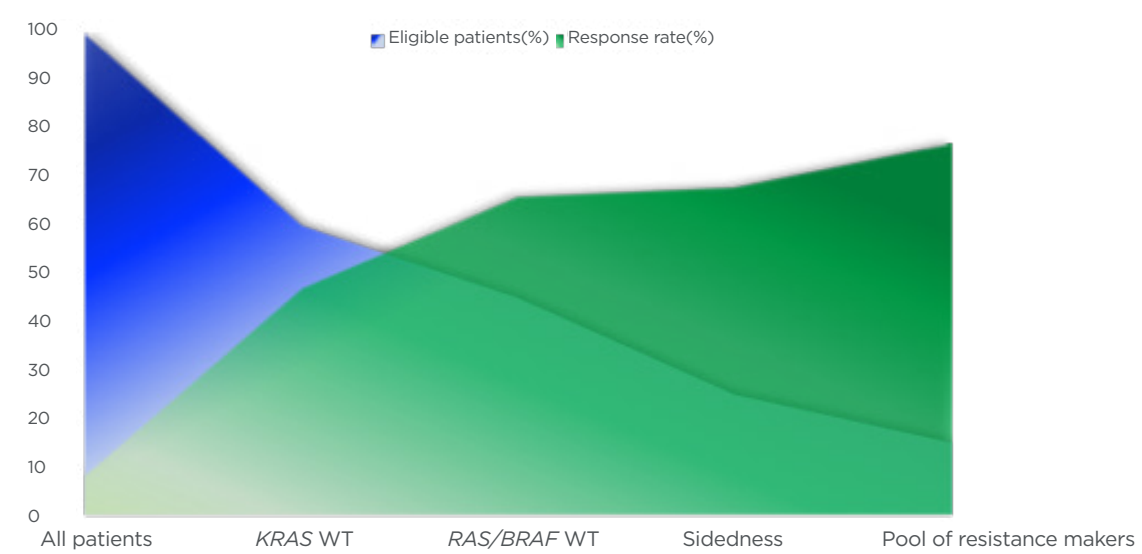

Figure 1: Negative hyper-selection of patients with metastatic colorectal cancer to anti-epidermal growth factor receptor monoclonal antibodies.

The graph shows the ascending response rate ( $\mathrm{y}$-axis, in green) of anti-EGFR therapy in mCRC based on patient selection by biomarkers (RAS and BRAF), sidedness, and a pool of resistance markers such as BRAF, PI3KCA, HER2, MET, PTEN, and AKT1 abnormalities, as well as NTRK/ROS1/ALK/RET rearrangements, which lead to a descending rate of eligible patients for targeted therapy ( $x$ axis, in blue). Since there are no predictive biomarkers of sensitivity for anti-EGFR therapy, the patient selection based on predictive markers of resistance may be denominated as negative hyper-selection.

EGRF: epidermal growth factor receptor; mCRC: metastatic colorectal cancer; WT: wild-type.

Hence, the estimated rate of patients with mCRC who are actually sensitive to cetuximab or panitumumab is lower than approximately $15 \%{ }^{9}$ The current recommendation to use anti-EGFR monoclonal antibodies in patients with left-sided tumours and RAS/BRAF wild-type status still is an incipient and imprecise clinical applicability of precision medicine in the systemic therapy of CRC. Therefore, the identification of refined biomarkers and novel targeted therapies are urgently needed (Figure 2; Table 1).

\section{NOVEL THERAPEUTIC TARGETS IN COLORECTAL CANCER}

\section{MSI-H CRC}

It is estimated that approximately $5 \%$ of patients with $\mathrm{mCRC}$ harbour high-frequency microsatellite instability (MSI-H), which might originate from two mechanisms: somatic hypermethylation of the $M L H 1$ gene promoter, commonly associated with BRAF V6OOE mutation; or point mutation of one of the mismatch repair genes, mainly MLH1 and MSH2.12,13 Patients with MSI-H CRC compose a subgroup with distinct molecular and clinical characteristics. Typically, they present a younger median age at diagnosis, with tumours predominantly located at the proximal colon, commonly with lymphocyte infiltration, and with a higher median number of tumour mutational burden (TMB). ${ }^{13}$ In addition, they have lower sensitivity to chemotherapy compared with patients classified as microsatellite stable (MSS), and, more importantly, they tend to be sensitive to immunotherapeutic approaches, such as immune checkpoint inhibitors (ICls).

Encouraging data from Phase I and || clinical trials $^{14-19}$ prompted the conception of the Phase III KEYNOTE-177 study, which compared the efficacy of standard chemotherapy (doublets plus anti-vascular endothelial growth factor or anti-EGFR) with pembrolizumab in 307 treatment-naïve patients with $\mathrm{MSI}-\mathrm{H}$ mCRC. ${ }^{20}$ One of the primary endpoints, progressionfree survival, was met: 8.2 months in the chemotherapy group versus 16.5 months in the immunotherapy group (hazard ratio [HR]: 0.60; 95\% confidence interval [Cl]: 0.45-0.80; $\mathrm{p}=0.0002)$. Likewise, the overall response rate (ORR) was statistically higher in the immunotherapy arm: $33.1 \%$ versus $43.8 \%$. 


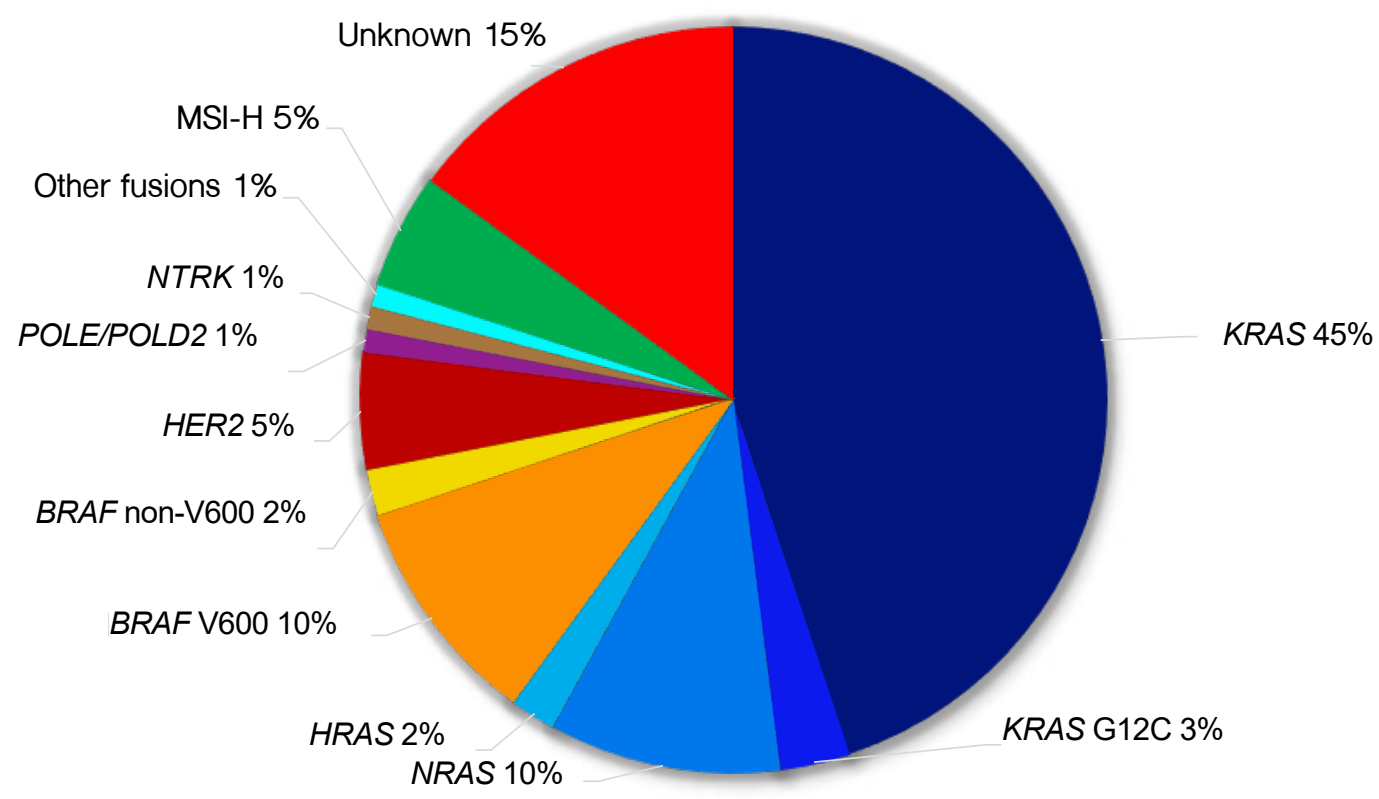

Figure 2: Molecular alterations with therapeutic implications in metastatic colorectal cancer.

MSI-H: high-frequency microsatellite instability.

Table 1: Predictive biomarkers and targeted therapies in metastatic CRC.

\begin{tabular}{|l|l|l|}
\hline Biomarkers & Rates* & Predictive role to systemic therapy \\
\hline KRAS non-G12C & $45 \%$ & Resistance to anti-EGFR therapy \\
\hline KRAS G12C & $3 \%$ & $\begin{array}{l}\text { Resistance to anti-EGFR therapy } \\
\text { Poor sensitivity to sotorasib or adagrasib alone }\end{array}$ \\
\hline NRAS & $10 \%$ & Resistance to anti-EGFR therapy \\
\hline HRAS & $2 \%$ & Resistance to anti-EGFR therapy \\
\hline BRAF V6OOE & $10 \%$ & $\begin{array}{l}\text { Sensitivity to encorafenib+cetuximab } \pm \text { binimetinib } \\
\text { Resistance to anti-EGFR therapy }\end{array}$ \\
\hline BRAF non-V6OOE & $2 \%$ & Uncertain \\
\hline HER2 amplification & $5 \%$ & $\begin{array}{l}\text { Sensitivity to anti-HER2 therapies } \\
\text { Potential resistance to anti-EGFR therapy }\end{array}$ \\
\hline NTRK fusions & $1 \%$ & Sensitivity to larotrectinib or entrectinib \\
\hline ALK/ROS1 fusions & $<1 \%$ & Sensitivity to ALK/ROS1 inhibitors \\
\hline MSI-H & $5 \%$ & Sensitivity to immune checkpoint inhibitors \\
\hline POLE/POLD2 & $1 \%$ & Potential sensitivity to immune checkpoint inhibitors \\
\hline
\end{tabular}

\section{${ }^{*}$ Approximate rates.}

MSI-H: high-frequency microsatellite instability, EGFR: epidermal growth factor receptor. 
Interestingly, $29.4 \%$ of the patients in the pembrolizumab group presented progressive disease compared with $12.3 \%$ in the chemotherapy group, predominantly in the first 4 months of treatment. Of the patients who presented objective response to immunotherapy, an impressive rate of $83 \%$ had ongoing responses at 24 months, compared with only $35 \%$ in the chemotherapy group. Another primary endpoint, OS, did not have mature data to be analysed.

$\mathrm{MSI}-\mathrm{H}$ is not the only biomarker to explain sensitivity to $\mathrm{ICls}$. Patients with $\mathrm{MSI}-\mathrm{H}$ who present with low TMB seem to have a lower probability to respond to immunotherapy. ${ }^{21} \mathrm{~A}$ high concordance rate is expected between MSI-H and TMB. Patients with abnormalities in DNA mismatch repair pathways, whether germline or somatic, tend to present higher number of nonsynonymous mutations, and thereby high TMB. In a study with 6,004 patients with $\mathrm{mCRC}$, 5\% were classified as MSI-H and 95\% as MSS. ${ }^{22}$ The median TMB was significantly higher in the population with MSI-H: 46.8 mutations/Mb versus 3.6 mutations/ $\mathrm{Mb}$. The median TMB in the overall population was 4.5 mutations/Mb. Approximately $3 \%$ of patients with MSS were classified as high TMB, defined as $\geq 12$ mutations/Mb. Variants in the mismatch repair genes, such as $M L H 1, M S H 2$, and MSH6, as well as in POLE, were significantly more common in this population of patients with high TMB and MSS relative to those with low TMB and MSS. ${ }^{22}$

\section{POLE}

POLE encodes the catalytic subunit of DNA polymerase $\varepsilon$, which acts in the replication of the DNA strand before cell division. ${ }^{23}$ POLE proofreading is an essential step in the maintenance of the integrity of the genome, which is consistent with the finding of ultra-mutated tumours in the presence of pathogenic exonuclease domain mutations, with a mean tumour mutational burden $>200$ mutations/Mb. ${ }^{24,25}$ POLE mutation is rarely found in malignancies, being identified in approximately $5-10 \%$ of endometrial cancer, ${ }^{26}$ $1 \%$ of $C R C,{ }^{27}$ and less frequently in gastric and pancreatic cancers. ${ }^{28}$

POLE-mutated CRC portends a better prognosis. In a population of 6,517 patients with CRC, 1\% (66 patients) harboured the mutation, which was associated with a reduced risk of recurrence and a superior OS in a population of patients with Stage II/III CRC. ${ }^{23}$ Patients with POLE-mutated CRC were younger at diagnosis, predominantly male, with a higher frequency of right-sided tumours, and with disease diagnosed at earlier stages compared with the wild-type counterparts..$^{23,25,29}$ They also demonstrated increased CD8+ Iymphocyte infiltration and expression of cytotoxic T-cell markers. ${ }^{23}$

This immunogenic subset of CRC has been demonstrated to be highly sensitive to the $\mathrm{ICls}$. Case reports with successful experiences in the treatment of metastatic CRC have been presented in the past few years. ${ }^{31,32}$ Since there is a U.S. Food and Drug Administration (FDA) approval for the use of pembrolizumab for patients with metastatic disease and $\mathrm{TMB}>10$ mutations/Mb, ${ }^{32}$ the use of immunotherapy should be strongly considered in the treatment of patients with POLE-mutated $\mathrm{mCRC}$. The probability to identify these mutations is higher in early-onset CRC compared to the late-onset. ${ }^{33}$

\section{$B R A F$}

$B R A F$ V600E mutation is found in approximately $10 \%$ of patients with $\mathrm{mCRC}{ }^{34-37}$ These patients have a poorer prognosis compared with the wild-type counterparts, demonstrating lower sensitivity to the standard chemotherapeutic drugs used in CRC, with lower ORR, and shorter progression-free survival and OS. ${ }^{38-40}$ More commonly found in right-sided tumours, this mutation, similarly to RAS mutations, also denotes resistance to the anti-EGFR monoclonal antibodies. ${ }^{41,42}$

The success of BRAF inhibitors in the systemic therapy of BRAF-mutated melanoma prompted the evaluation of these drugs in $\mathrm{MCRC}$. However, Phase I data addressing the efficacy of vemurafenib in patients with BRAF-mutated $\mathrm{mCRC}$ showed poor efficacy. ${ }^{43}$ Preclinical studies demonstrated that BRAF inhibition induced adaptive feedback reactivation of mitogenactivated protein kinase signalling, often mediated by EGFR activation, suggesting that the combination of BRAF inhibitor with an antiEGFR monoclonal antibody might overcome this 
therapeutic resistance. ${ }^{44-46}$ Subsequently, a Phase IB study confirmed the hypothesis, demonstrating that the combination of vemurafenib, irinotecan, and cetuximab yielded $35 \%$ of ORR in a population of 19 patients with BRAF-mutated mCRC. ${ }^{47}$ Additionally, further work demonstrated the clinical activity of the combination of BRAF and EGFR inhibition with or without mitogenactivated protein kinase kinase (MEK) inhibition in BRAF-mutated mCRC. ${ }^{44}$

These promising findings elicited the conception of the Phase III BEACON study, a three-arm clinical trial that explored the combination of BRAF and EGFR inhibition with MEK inhibition. A total of 665 patients with BRAF V600E-mutated $\mathrm{MCRC}$ who had been submitted to at least one previous line of systemic therapy were randomised to one of three arms: the triplet-regimen composed of encorafenib plus binimetinib plus cetuximab; the doublet-regimen with encorafenib plus cetuximab; and the control arm with irinotecanbased regimens (folinic acid, fluorouracil, and irinotecan; or irinotecan) plus cetuximab. ${ }^{48}$ The primary end points were OS and ORR in the triplet-regimen arm compared to the control arm. Updated survival results showed a median OS of 9.3 months in the triplet arm versus 5.9 months in the control arm (HR: 0.60; 95\% Cl: 0.47-0.75). ${ }^{49}$ The ORR was $27 \%, 20 \%$, and $2 \%$ in the triplet, doublet, and control arms, respectively. A comparison of the median OS in the doublet arm (9.3 months) with the control arm, a secondary endpoint, also favoured the BRAF inhibitor (HR: 0.61; 95\% Cl: 0.48-0.77). There was no statistically significant difference between the triplet and doublet arms in OS: 9.3 months in both groups (HR: 0.95; 95\% Cl: 0.74-1.21). ${ }^{49}$ Grade $\geq 3$ adverse events were found in $66 \%, 58 \%$, and $64 \%$ of the patients in the triplet, doublet, and control arms, respectively. Based on BEACON data, the FDA has approved the combination of encorafenib plus cetuximab for the treatment of patients with $\mathrm{MCRC}$ and a BRAF V6OOE mutation with at least one prior systemic therapy. ${ }^{50}$

The clinical relevance of non-V600 BRAF mutations has not yet been fully elucidated. These mutations have been found in approximately $2 \%$ of patients with mCRC, of which the D594N (Class III) and G469A (Class II) mutations seem to be the most frequent. ${ }^{51}$
The patients who harbour these atypical BRAF mutations seem to present similar prognosis compared to the wild-type counterparts. Unlike V600E, these atypical mutations are mostly identified in left-sided tumours, and younger male patients. ${ }^{51}$ In addition, most of them are MSS and RAS mutations are not mutually exclusive in this context, occurring in approximately one-third of non-V600 patients. ${ }^{51,52}$ The predictive value of these mutations for the deployment of anti-EGFR monoclonal antibodies is not yet entirely clear, and appears to differ according to the underlying $B R A F$ class. Class II mutations appear to be resistant while Class III are sensitive, although with limited duration. ${ }^{53-55}$ Furthermore, non-V600 $B R A F$ mutations might be involved in the development of adaptive resistance to EGFR inhibition. ${ }^{51}$ These distinct class-specific biochemical and functional properties highlight the importance to decipher the unique biology of atypical BRAF mutations in order to promote novel clinical trial design and ultimately offer effective therapeutic options for patients.

\section{KRAS G12C}

KRAS mutations are the most common activating genetic mutations in solid tumours, mainly in pancreatic cancer, non-small cell lung cancer, and CRC, where they are estimated to be found in approximately $45 \%$ of tumours. ${ }^{37,56,57}$ Right-sided tumours present a higher percentage of KRAS mutations compared with their left-sided counterparts, mainly in the cecum, where approximately $70 \%$ of the tumours harbour the mutation. ${ }^{56}$ For decades, KRAS mutations have not been deemed as actionable, but, together with other RAS mutations, they predict resistance to anti-EGFR monoclonal antibodies in CRC. ${ }^{58,59}$

Despite years of research focus, targeting KRAS has been an elusive goal in cancer therapy since the mutated protein has high affinity for guanosine triphosphate or guanosine diphosphate and has no binding pocket. 60 In addition, inhibition of the downstream effectors in the mitogen-activated protein kinase pathway (BRAF-MEK-ERK) has proven ineffective in clinical trials. ${ }^{60}$

The codons 12 and 13 in exon 2 are the most commonly altered in KRAS mutations, occurring 
in approximately $30 \%$ and $10 \%$, respectively, of the patients with $\mathrm{mCRC} .^{57}$ The amino acid changes p.G12D, p.G12V, and p.G13D are the most frequent of these codons in CRC, found in approximately $13 \%, 10 \%$, and $9 \%$ of the patients, respectively. ${ }^{57}$ The oncoprotein KRAS p.G12C is found in $1-3 \%$ of patients with mCRC. 57,61 The substitution of glycine for cysteine at position 12 results in a predominantly guanosine triphosphatebound KRAS protein, the active form, favouring proliferation and survival of tumour cells. ${ }^{62,63}$

Recently, the isoform KRAS G12C has demonstrated to be targetable by a covalent allele-specific inhibitor. Sotorasib (AMG510) is a small molecule that specifically and irreversibly inhibits KRAS G12C in its inactive guanosine diphosphate-bound state through an interaction with one of its pockets. ${ }^{61}$ It was evaluated in a Phase I trial with 129 previously treated patients with advanced solid tumours harbouring the KRAS G12C mutation. ${ }^{61}$ In the overall trial population, of the 42 patients with CRC, only $3(7 \%)$ presented partial response, but 28 (67\%) experienced stable disease. On the other hand, $32 \%$ of the 59 patients with non-small cell lung cancer had partial response, and $56 \%$ showed stable disease. Adagrasib (MRTX849) is another KRAS G12C inhibitor under therapeutic development and it has shown promising efficacy in preclinical studies and preliminary clinical findings. ${ }^{64}$ Additional Phase I/II clinical trials are currently evaluating the efficacy of adagrasib in KRAS G12C-mutated malignancies, and an ongoing Phase III clinical trial is comparing the efficacy of adagrasib in combination with cetuximab versus chemotherapy in the second-line setting for patients with $\mathrm{MCRC}$ and KRAS G12C mutation (NCTO4793958). ${ }^{65}$

Interestingly, patients with KRAS G12C-mutated $\mathrm{mCRC}$ seem to present poorer clinical outcomes compared with the patients with KRAS non-G12C mutations. ${ }^{66,67}$ A recent singleinstitutional study identified 187 patients with KRAS G12C from an original population of 4,685 patients with $\mathrm{mCRC} .{ }^{66}$ When compared to a cohort of 720 patients with KRAS non-G12C mutations, these 187 patients had shorter OS, excluding patients who had undergone metastasectomy: 21.2 months versus 31.6 months $(p=0.003)$. Another cohort of 839 patients with $\mathrm{mCRC}$ also found an inferior OS in G12C population compared with the non-G12C: 25.9 months versus 35.8 months (HR: 1.55; 95\% Cl: 1.08-2.24; $p=0.018$ ), which was confirmed by multivariate analysis (HR: $1.81 ; \quad 95 \% \quad \mathrm{Cl}: 1.20-2.70 ; \quad \mathrm{p}=0.04) .{ }^{67}$ Correlative findings also demonstrated that this subgroup of patients with $\mathrm{mCRC}$ show a distinct mutational profile, with higher rates of $A P C$ co-mutations compared with the patients without the G12C mutation, but lower rates of $B R A F, E R B B 4, N R A S$, and TP53 co-mutations.

The reasons for the different efficacy of the KRAS G12C inhibitor according to the tissue of origin are not clear. ${ }^{61}$ Ongoing translational studies will be crucial in the understanding of the probable intrinsic resistance of KRAS G12C-mutated mCRC to the KRAS G12C inhibitors as monotherapy, and for the design of clinical trials evaluating the combination of these inhibitors with other therapeutic strategies.

\section{HER2}

Comprehensive molecular characterisation of CRC and the greater availability of nextgeneration sequencing in tumour genomic profiling have demonstrated that HER2 amplification is found in approximately $5-10 \%$ of patients with RAS wild-type mCRC.7,8,27 This molecular abnormality is predominantly identified in patients with RAS/BRAF wild-type patients, who may harbour HER2 amplification primarily or secondarily as a mechanism of resistance to anti-EGFR therapy. Exploratory analyses suggest that patients with RAS wild-type who harbour HER2 amplification derive lower, if any, benefit from anti-EGFR therapy.7,8 Based on the successful experiences of anti-HER2 therapy in HER2amplified breast and gastric cancers, the identification of this molecular abnormality in mCRC prompted the evaluation of anti-HER2 therapy in clinical trials.

HERACLES was the first clinical trial addressing the efficacy of anti-HER2 therapy in HER2positive mCRC.71 This proof-of-concept Phase II study was comprised of 27 treatmentrefractory patients, of which 30\% presented an objective response to the combination of trastuzumab plus lapatinib, and an additional $44 \%$ had stable disease. The subsequent MyPathway Phase II study showed that $32 \%$ of the 57 heavily pre-treated patients with HER2positive $\mathrm{mCRC}$ had objective response to the 
combination of trastuzumab plus pertuzumab. ${ }^{72}$ The Phase II TAPUR basket trial evaluated the same combination of anti-HER2 therapy in 28 previously treated patients with HER2-positive $\mathrm{mCRC}$, and $14 \%$ of the patients demonstrated objective response. ${ }^{73}$ Impressive findings were demonstrated by the preliminary results of the Phase II MOUNTAINEER study, which evaluated the combination of tucatinib plus trastuzumab. Of the 22 evaluable previously treated patients with HER2-positive mCRC, 55\% presented objective response. ${ }^{74}$ Likewise, promising data have also been presented by DESTINY-CRCO1 trial, which showed $45 \%$ of ORR with trastuzumab deruxtecan in 53 patients with previously treated HER2-positive $\mathrm{mCRC}^{75}$ Taken together, these initial clinical trials have demonstrated that HER2 is a viable therapeutic target in $\mathrm{MCRC}$, with encouraging efficacy data of the dual anti-HER2 therapy in treatment refractory patients. However, the FDA has not yet approved anti-HER2 therapies for $\mathrm{mCRC}$ in the USA. The results of the ongoing randomised clinical trial SWOG1613 evaluating the combination of trastuzumab plus pertuzumab in RAS/BRAF wild-type patients are eagerly awaited (NCT03365882), ${ }^{76}$ as well as the results of the Phase II DESTINY-CRCO2 trial, with more data of trastuzumab deruxtecan in $\mathrm{MCRC}$ (NCT04744831). ${ }^{77}$

\section{NTRK, ALK, and ROS1}

NTRK are genes that encode the tropomyosin receptor kinase (Trk) family, which is comprised of three transmembrane proteins, TrkA, TrkB, and TrkC receptors, which are encoded by the NTRK1, NTRK2, and NTRK3 genes, respectively.78 The signal transduction pathways activated by these receptors are associated with proliferation, differentiation, and survival in normal and neoplastic neuronal cells. ${ }^{79}$ Gene fusions of the NTRK are the main molecular abnormalities with known oncogenic and transforming potential. ${ }^{80}$
Based on a study with 408 patients with CRC, it is estimated a prevalence rate of $0.5 \%$ of this gene fusion. ${ }^{81}$ Efficacy of larotrectinib in NTRK fusionpositive $\mathrm{mCRC}$ was demonstrated in a basket trial with 55 patients with solid malignancies, of which four had mCRC. ${ }^{82}$ Three patients presented tumour shrinkage and one had stable disease. Entrectinib, a pan-Trk inhibitor, has also been demonstrated to be effective in this subset of patients. ${ }^{83}$ Other gene fusions, such as those involving $A L K$ and $R O S 1$, are rarely found in $\mathrm{mCRC}$, but, once present, they portend a poorer prognosis. ${ }^{84,85}$ Targeted therapies, including entrectinib, have been effective in this subgroup of patients with mCRC. ${ }^{86}$

\section{CONCLUSIONS}

Meaningful precision-based advancements in the therapeutic options for $\mathrm{MCRC}$ have been challenging and slow to realisation. Comprehensive molecular profiling and circulating tumour DNA highlights a markedly heterogeneous disease at the genomic, epigenomic, and transcriptomic levels; however, to date, they only reflect a low frequency of actionable alterations. For almost two decades, clinical applicability of precision oncology in MCRC was limited to the identification of RAS mutations as predictive biomarkers of resistance to the use of anti-EGFR monoclonal antibodies. However, novel therapeutic targets have emerged in recent years, refining the landscape of systemic therapy of the disease. The benefit of $\mathrm{ICls}$ in patients with $\mathrm{MSI}-\mathrm{H}$ and in those with POLE mutations or high TMB, the combination of BRAF with EGFR inhibition in patients with BRAF V600 mutations, the advent of allele-specific KRAS G12C inhibitors, and the promising findings of dual anti-HER2 therapy in HER2-positive MCRC cases have ushered in a new era of precision oncology for $\mathrm{MCRC}$, providing personalised treatments and sustaining hope for patients affected by this disease.

\section{References}

1. Siegel RL et al. Cancer statistics, 2020 CA Cancer J Clin. 2020;70(1):7-30.

2. Noone Aet al. SEER cancer statistics, 1975-2015. 2018. Available at: https://seer.cancer.gov/archive/ csr/1975_2015/. Last accessed: 26 august 2021.
3. Siegel RL et al. Colorectal cancer statistics, 2020. CA Cancer J Clin. 2020;70(3):145-64

4. Morano F et al. Negative hyperselection of patients with RAS and BRAF wild-type metastatic colorectal cancer who received panitumumab-based maintenance therapy. J Clin Oncol. 2019;37(33):3099-110.

5. Di Nicolantonio $F$ et al. Wild-type $B R A F$ is required for response to panitumumab or cetuximab in metastatic colorectal cancer. J Clin Oncol. 2008;26(35):5705-12. 
6. Loupakis F et al. KRAS codon 61, 146 and $B R A F$ mutations predict resistance to cetuximab plus irinotecan in KRAS codon 12 and 13 wild-type metastatic colorectal cancer Br J Cancer. 2009;101(4):715-21.

7. Raghav $\mathrm{K}$ et al. Validation of HER2 amplification as a predictive biomarker for anti-epidermal growth factor receptor antibody therapy in metastatic colorectal cancer. JCO Prec Oncol. 2019;3:1-13.

8. Martin $\mathrm{V}$ et al. HER2 gene copy number status may influence clinical efficacy to anti-EGFR monoclonal antibodies in metastatic colorectal cancer patients. $\mathrm{Br} \mathrm{J}$ Cancer. 2013;108(3):668-75.

9. Bardelli A, Siena S. Molecular mechanisms of resistance to cetuximab and panitumumab in colorectal cancer. J Clin Oncol. 2010;28(7):1254-61.

10. Venook AP et al. Effect of firstline chemotherapy combined with cetuximab or bevacizumab on overall survival in patients with KRAS wild-type advanced or metastatic colorectal cancer: a randomized clinical trial. JAMA. 2017;317(23):2392-401.

11. Arnold D et al. Prognostic and predictive value of primary tumour side in patients with RAS wild-type metastatic colorectal cancer treated with chemotherapy and EGFR directed antibodies in six randomized trials. Ann Oncol. 2017;28(8):1713-29.

12. Boland $\mathrm{CR}$ et al. A National Cancer Institute Workshop on microsatellite instability for cancer detection and familial predisposition: development of international criteria for the determination of microsatellite instability in colorectal cancer. Cancer Res. 1998;58(22):5248-57.

13. Sinicrope FA. Lynch Syndromeassociated colorectal cancer. N Engl J Med. 2018;379(8):764-73.

14. Overman MJ et al. Durable clinical benefit with nivolumab plus ipilimumab in DNA mismatch repairdeficient/microsatellite instabilityhigh metastatic colorectal cancer. J Clin Oncol. 2018;36(8):773-79.

15. Overman MJ et al. Nivolumab in patients with metastatic DNA mismatch repair-deficient or microsatellite instability-high colorectal cancer (CheckMate 142): an open-label, multicentre, Phase 2 study. Lancet Oncol. 2017;18(9):1182-91.

16. Overman MJ et al. Nivolumab \pm ipilimumab in treatment (tx) of patients (pts) with metastatic colorectal cancer (mCRC) with and without high microsatellite instability (MSI-H): CheckMate-142 interim results. J Clin Oncol. 2016;34(Suppl 15):3501.

17. Le DT et al. PD-1 blockade in tumors with mismatch-repair deficiency. $\mathrm{N}$ Engl J Med. 2015;372(26):2509-20.
18. Brahmer JR et al. Safety and activity of anti-PD-L1 antibody in patients with advanced cancer. N Engl J Med. 2012;366(26):2455-65.

19. Topalian SL et al. Safety, activity, and immune correlates of anti-PD-1 antibody in cancer. N Engl J Med. 2012;366(26):2443-54.

20. Andre $T$ et al. Pembrolizumab versus chemotherapy for microsatellite instability-high/mismatch repair deficient metastatic colorectal cancer: the Phase 3 KEYNOTE-177 Study. J clin Oncol. 2020;38(Suppl 18):LBA4

21. Schrock $A B$ et al. Tumor mutational burden is predictive of response to immune checkpoint inhibitors in MSIhigh metastatic colorectal cancer. Ann Oncol. Jul 2019;30(7):1096-103.

22. Fabrizio DA et al. Beyond microsatellite testing: assessment of tumor mutational burden identifies subsets of colorectal cancer who may respond to immune checkpoint inhibition. J Gastrointest Oncol. 2018;9(4):610-17.

23. Domingo $\mathrm{E}$ et al. Somatic POLE proofreading domain mutation, immune response, and prognosis in colorectal cancer: a retrospective pooled biomarker study. Lancet Gastroenterol Hepatol. 2016;1(3):207-16

24. Pursell ZF. Yeast DNA polymerase $\varepsilon$ participates in leading-strand DNA replication. Science. 2007;317(5834):127-30.

25. $\mathrm{Hu} \mathrm{H}$ et al. Ultra-mutated colorectal cancer patients with POLE driver mutations exhibit distinct clinical patterns. Cancer Med. 2021;10(1):135-42.

26. Levine DA. Integrated genomic characterization of endometrial carcinoma. Nature. 2013;497(7447):67-73.

27. Kandoth $\mathrm{C}$ et al.; Cancer Genome Atlas Research Network. Comprehensive molecular characterization of human colon and rectal cancer. Nature. 2012;487(7407):330-7.

28. Rayner $E$ et al. A panoply of errors: polymerase proofreading domain mutations in cancer. Nat Rev Cancer. 2016;16(2):71-81.

29. Hino $\mathrm{H}$ et al. Clinicopathological and mutational analyses of colorectal cancer with mutations in the POLE gene. Cancer Med. 2019;8(10):4587-97.

30. Gong J et al. Response to PD-1 blockade in microsatellite stable metastatic colorectal cancer harboring a POLE mutation. J Natl Compr Canc Netw. 2017;15(2):142-7.

31. Silberman $\mathrm{R}$ et al. Complete and prolonged response to immune checkpoint blockade in POLEmutated colorectal cancer. JCO Precis Oncol. 2019;3:1-5.

32. Merck \& Co., Inc. Highlights of prescribing information for Keytruda (pembrolizumab) injection. 2014 Available at: https://www.merck.com/ product/usa/pi_circulars/k/keytruda/ keytruda pi.pdf. Last accessed: 26 August 2021

33. Ahn S-M et al. The somatic POLE P286R mutation defines a unique subclass of colorectal cancer featuring hypermutation, representing a potential genomic biomarker for immunotherapy. Oncotarget. 2016;7(42):68638-49.

34. Yaeger $\mathrm{R}$ et al. Clinical sequencing defines the genomic landscape of metastatic colorectal cancer. Cancer Cell. 2018;33(1):125-36.

35. Barras D et al. BRAF V6OOE mutant colorectal cancer subtypes based on gene expression. Clin Cancer Res. 2017;23(1):104-15

36. Bylsma LC et al. Prevalence of RAS and BRAF mutations in metastatic colorectal cancer patients by tumor sidedness: a systematic review and meta-analysis. Cancer Med. 2020;9(3):1044-57

37. AACR Project GENIE Consortium. AACR Project Genie: powering precision medicine through an international consortium. Cancer Discov. 2017;7(8):818-31.

38. Clarke CN, Kopetz ES. BRAF mutant colorectal cancer as a distinct subset of colorectal cancer: clinical characteristics, clinical behavior, and response to targeted therapies. J Gastrointest Oncol. 2015;6(6):660-7.

39. Tran B et al. Impact of BRAF mutation and microsatellite instability on the pattern of metastatic spread and prognosis in metastatic colorectal cancer. Cancer. 2011;117(20):4623-32.

40. Loupakis F et al. Initial therapy with FOLFOXIRI and bevacizumab for metastatic colorectal cancer. N Engl J Med. 2014;371(17):1609-18.

41. Douillard JY et al. PanitumumabFOLFOX4 treatment and RAS mutations in colorectal cancer. $\mathrm{N}$ Engl J Med. 2013;369(11):1023-34.

42. Pietrantonio $\mathrm{F}$ et al. Predictive role of BRAF mutations in patients with advanced colorectal cancer receiving cetuximab and panitumumab: a meta-analysis. Eur J Cancer 2015;51(5):587-94.

43. Kopetz S et al. Phase II pilot study of vemurafenib in patients with metastatic BRAF-mutated colorectal cancer. J Clin Oncol. 2015;33(34):4032-8.

44. Corcoran RB et al. Combined BRAF, EGFR, and MEK inhibition in patients with $B R A F^{V 600 E}$-mutant colorectal cancer. Cancer Discov. 2018;8(4):428-43.

45. Prahallad A et al. Unresponsiveness of colon cancer to BRAF (V600E) inhibition through feedback activation of EGFR. Nature. 2012;483(7387):100-3. 
46. Mao M et al. Resistance to BRAF inhibition in BRAF-mutant colon cancer can be overcome with PI3K inhibition or demethylating agents. Clin Cancer Res. 2013;19(3):657-67.

47. Hong DS et al. Phase IB study of vemurafenib in combination with irinotecan and cetuximab in patients with metastatic colorectal cancer with BRAFV600E mutation. Cancer Discov. 2016;6(12):1352-65.

48. Kopetz $S$ et al. Encorafenib, binimetinib, and cetuximab in $B R A F$ V600E-mutated colorectal cancer. N Engl J Med. 2019;381(17):1632-43.

49. Kopetz S et al. Encorafenib plus cetuximab with or without binimetinib for BRAF V6O0E metastatic colorectal cancer: Updated survival results from a randomized, three-arm, Phase III study versus choice of either irinotecan or FOLFIRI plus cetuximab (BEACON CRC). J Clin Oncol. 2020;38(Suppl 4):8.

50. Braftovi. (encorafenib). Array BioPharma Inc. Boulder. CO.202O.

51. Johnson B et al. Atypical, non-V600 BRAF mutations as a potential mechanism of resistance to EGFR Inhibition in metastatic colorectal cancer. JCO Precis Oncol. 2019;3:1-10.

52. Kotani $D$ et al. Clinicopathological features, efficacy of anti-EGFR therapy, and survival outcomes in patients with BRAF non-V600 mutated metastatic colorectal cancer J Clin Oncol. 2019;37(Suppl 4):659.

53. Fontana E, Valeri N. Class(y) dissection of BRAF heterogeneity: beyond non-V600. Clin Cancer Res. 2019;25(23):6896-8.

54. Wang $Y$ et al. Activity of EGFR antibody in non-V600 BRAF mutant metastatic colorectal cancer. Ann Oncol. 2019;30(1):147-49.

55. Yaeger R et al. Response to antiEGFR therapy in patients with BRAF non-V600-mutant metastatic colorectal cancer. Clin Cancer Res. 2019;25(23):7089-97.

56. Loree JM et al. Classifying colorectal cancer by tumor location rather than sidedness highlights a continuum in mutation profiles and consensus molecular subtypes. Clin Cancer Res. 2018:24(5):1062-72.

57. Vaughn $C P$ et al. Frequency of KRAS, BRAF, and NRAS mutations in colorectal cancer Genes Chromosomes Cancer. 2011;50(5):307-12

58. Misale $\mathrm{S}$ et al. Emergence of KRAS mutations and acquired resistance to anti-EGFR therapy in colorectal cancer. Nature. 2012;486(7404):532-6.

59. Sorich $M$ et al. Extended RAS mutations and anti-EGFR monoclonal antibody survival benefit in metastatic colorectal cancer: a metaanalysis of randomized, controlled trials. Ann Oncol. 2015;26(1):13-21.

60. Moore AR et al. RAS-targeted therapies: is the undruggable drugged? Nat Rev Drug Discov. 2020;19(8):533-52

61. Hong DS et al. KRAS ${ }^{\mathrm{G} 12 \mathrm{C}}$ Inhibition with sotorasib in advanced solid tumors. N Engl J Med. 2020;383(13):1207-17.

62. Ostrem JM et al. K-Ras(G12C) inhibitors allosterically control GTP affinity and effector interactions Nature. 2013;503(7477):548-51.

63. Kargbo RB. Inhibitors of G12C mutant Ras proteins for the treatment of cancers. ACS Med Chem Lett; 2018;10(1):10-11.

64. Hallin $\mathrm{J}$ et al. The KRAS ${ }^{\mathrm{G} 12 \mathrm{C}}$ inhibitor MRTX849 provides insight toward therapeutic susceptibility of KRASmutant cancers in mouse models and patients. Cancer Discov. 2020;10(1):54-71.

65. Mirati Therapeutics Inc. Phase 3 study of MRTX849 with cetuximab vs chemotherapy in patients with advanced colorectal cancer with KRAS G12C mutation (KRYSTAL-10) NCTO4793958. https://clinicaltrials. gov/ct2/show/NCT04793958.

66. Henry JT et al. Comprehensive clinical and molecular characterization of KRAS $^{\mathrm{G} 12 \mathrm{C}}$-mutant colorectal cancer. JCO Precis Oncol. 2021:5:613-21.

67. Schirripa $M$ et al. KRAS G12C metastatic colorectal cancer: specific features of a new emerging target population. Clin Colorectal Cancer. 2020;19(3):219-25.

68. Pietrantonio F et al. Heterogeneity of acquired resistance to anti-EGFR monoclonal antibodies in patients with metastatic colorectal cancer. Clin Cancer Res. 2017;23(10):2414-22.

69. Diaz Jr LA et al. The molecular evolution of acquired resistance to targeted EGFR blockade in colorectal cancers. Nature. 2012;486(7404):537-40.

70. Misale $\mathrm{S}$ et al. Resistance to anti-EGFR therapy in colorectal cancer: from heterogeneity to convergent evolution. Cancer Discov. 2014;4(11):1269-80.

71. Sartore-Bianchi A et al. Dual-targeted therapy with trastuzumab and lapatinib in treatment-refractory, KRAS codon 12/13 wild-type, HER2positive metastatic colorectal cancer (HERACLES): a proof-of-concept, multicentre, open-label, Phase 2 trial. Lancet Oncol. 2016;17(6):738-46.

72. Meric-Bernstam F et al. Pertuzumab plus trastuzumab for HER2-amplified metastatic colorectal cancer (MyPathway): an updated report from a multicentre, open-label, Phase 2a, multiple basket study. Lancet Oncol. 2019;20(4):518-30.

73. Gupta R et al. Pertuzumab plus trastuzumab $(P+T)$ in patients
Pts) with colorectal cancer (CRC) with ERBB2 amplification or overexpression: results from the TAPUR Study. J Clin Oncol. 2020;38(Suppl 4):132

74. Strickler J et al. Trastuzumab and tucatinib for the treatment of HER2 amplified metastatic colorectal cancer (MCRC): initial results from the MOUNTAINEER trial. Ann Oncol. 2019;30(Suppl 5):v200.

75. Siena S et al. Trastuzumab deruxtecan (DS-8201) in patients with HER2-expressing metastatic colorectal cancer (DESTINY-CRCO1): a multicentre, open-label, Phase 2 trial. Lancet Oncol. 2021;22(6):779-89.

76. Southwest Oncology Group. S1613, trastuzumab and pertuzumab or cetuximab and irinotecan hydrochloride in treating patients with locally advanced or metastatic HER2/Neu amplified colorectal cancer that cannot be removed by surgery. NCT03365882. https:// clinicaltrials.gov/ct2/show/ NCT03365882.

77. Daiichi Sankyo, Inc. Trastuzumab deruxtecan in participants with HER2-overexpressing advanced or metastatic colorectal cancer (DESTINY-CRCO2). NCTO4744831. https://clinicaltrials.gov/ct2/show/ NCTO4744831.

78. Amatu A et al. NTRK gene fusions as novel targets of cancer therapy across multiple tumour types. ESMO Open. 2016;1(2):e000023.

79. Nakagawara A. Trk receptor tyrosine kinases: a bridge between cancer and neural development. Cancer Lett. 2001:169(2):107-14.

80. Vaishnavi Aet al. TRKing down an old oncogene in a new era of targeted therapy. Cancer Discov. 2015;5(1):25-34

81. Créancier $L$ et al. Chromosomal rearrangements involving the NTRK1 gene in colorectal carcinoma. Cancer Lett. 2015;365(1):107-11.

82. Drilon A et al. Efficacy of larotrectinib in TRK fusion-positive cancers in adults and children. $\mathrm{N}$ Engl J Med. 2018;378(8):731-9.

83. Sartore-Bianchi A et al. Sensitivity to entrectinib associated with a novel LMNA-NTRK1 gene fusion in metastatic colorectal cancer J Nat Cancer Inst. 2016;108(1):djv306.

84. Pietrantonio F et al. ALK, ROS1, and NTRK rearrangements in metastatic colorectal cancer. J Natl Cancer Inst. 2017;109(12):djx089.

85. Yakirevich E et al. Oncogenic ALK fusion in rare and aggressive subtype of colorectal adenocarcinoma as a potential therapeutic target. Clin Cancer Res. 2016;22(15):3831-40.

86. Amatu $\mathrm{A}$ et al. Novel CAD-ALK gene rearrangement is drugable by entrectinib in colorectal cancer. $\mathrm{Br} J$ Cancer. 2015:113(12):1730-4. 